PROCEEDINGS OF THE

AMERICAN MATHEMATICAL SOCIETY

Volume 130, Number 4, Pages 1095-1101

S 0002-9939(01)06250-5

Article electronically published on October 3, 2001

\title{
NEW PROOF OF THE COBORDISM INVARIANCE OF THE INDEX
}

\author{
MAXIM BRAVERMAN
}

(Communicated by Jozef Dodziuk)

\begin{abstract}
We give a simple proof of the cobordism invariance of the index of an elliptic operator. The proof is based on a study of a Witten-type deformation of an extension of the operator to a complete Riemannian manifold. One of the advantages of our approach is that it allows us to treat directly general elliptic operators which are not of Dirac type.
\end{abstract}

\section{INTRODUCTION}

Recently several simple proofs of the cobordism invariance of the index were established; cf. [4, [5. Th. 6.2], 6]. In this note we present still another proof of this fact. Unlike other authors we don't impose any restrictions on the dimension of the manifold and don't assume that our operator is of Dirac type.

1.1. The setting. Let $E^{+}, E^{-}$be Hermitian vector bundles over a closed Riemannian manifold $M$. Let $A^{+}: C^{\infty}\left(M, E^{+}\right) \rightarrow C^{\infty}\left(M, E^{-}\right)$be an elliptic pseudodifferential operator. Let $A^{-}: C^{\infty}\left(M, E^{-}\right) \rightarrow C^{\infty}\left(M, E^{+}\right)$be the formal adjoint of $A^{+}$and consider the operator

$$
A:=\left[\begin{array}{cc}
0 & A^{-} \\
A^{+} & 0
\end{array}\right]: C^{\infty}\left(M, E^{+} \oplus E^{-}\right) \rightarrow C^{\infty}\left(M, E^{+} \oplus E^{-}\right) .
$$

This operator is essentially self-adjoint and we denote by the same letter $A$ its extension to a self-adjoint operator acting on the space $L^{2}\left(M, E^{+} \oplus E^{-}\right)$of squareintegrable sections.

Suppose now that $M$ is a boundary of a Riemannian manifold $W$, which is isometric near the boundary to the cylinder $U=M \times(-\varepsilon, 0]$. Let $F$ be a Hermitian vector bundle over $W$, whose restriction to $U$ is isomorphic to the lift of $E^{+} \oplus E^{-}$.

Theorem 1.2. Assume that there exists a self-adjoint hypo-elliptic pseudo-differential operator $B: C^{\infty}(W, F) \rightarrow C^{\infty}(W, F)$, which near the boundary takes the form

$$
B=\gamma \frac{\partial}{\partial t}+A
$$

Received by the editors October 11, 2000.

1991 Mathematics Subject Classification. Primary 32L20; Secondary 58G10, 14F17.

Key words and phrases. Vanishing theorem, Clifford bundle, Dirac operator, AndreottiGrauert theorem, Melin inequality.

This research was partially supported by grant No. 96-00210/1 from the United States-Israel Binational Science Foundation (BSF). 
where $t$ is the normal coordinate and $\gamma$ is a skew-adjoint bundle map independent of $t$ such that $\left.\gamma\right|_{E^{ \pm}}= \pm \sqrt{-1}$. Then the index ind $A:=\operatorname{dim} \operatorname{Ker} A^{+}-\operatorname{dim} \operatorname{Ker} A^{-}=$ 0 .

1.3. The plan of the proof. Let $\tilde{W}$ denote the complete non-compact Riemannian manifold obtained from $W$ by attaching the semi-infinite cylinder $M \times[0, \infty)$ to the boundary. We extend the bundle $F$ and the operator $B$ to $\tilde{W}$ in the obvious way.

Consider the exterior algebra $\Lambda^{\bullet} \mathbb{C}=\Lambda^{0} \mathbb{C} \oplus \Lambda^{1} \mathbb{C}$. It has two (anti)-commuting actions $c_{L}$ and $c_{R}$ (left and right action) of the Clifford algebra of $\mathbb{R}$; cf. Subsection 2.1. Set $\tilde{F}=F \otimes \Lambda \bullet \mathbb{C}$ and consider the operator

$$
\tilde{B}:=\sqrt{-1} B \otimes c_{L}(1): C^{\infty}(\tilde{W}, \tilde{F}) \rightarrow C^{\infty}(\tilde{W}, \tilde{F}) .
$$

Let $p: \tilde{W} \rightarrow \mathbb{R}$ be a map whose restriction to $M \times(1, \infty)$ is the projection on the second factor, and such that $p(W)=0$ (see Subsection 2.1 for a convenient choice of this function). For any $a \in \mathbb{R}$, consider the operator $\mathbf{B}_{a}:=\tilde{B}-1 \otimes c_{R}((p(t)-a))$. Then (cf. Lemma 2.2)

$$
\mathbf{B}_{a}^{2}=B^{2} \otimes 1-R+|p(x)-a|^{2},
$$

where $R: \Gamma(\tilde{W}, \tilde{F}) \rightarrow \Gamma(\tilde{W}, \tilde{F})$ is a bounded operator.

Set ind $\mathbf{B}_{a}:=\operatorname{dim} \operatorname{Ker} \mathbf{B}_{a}^{+}-\operatorname{dim} \operatorname{Ker} \mathbf{B}_{a}^{-}$, where $\mathbf{B}_{a}^{ \pm}$denote the restriction of $\mathbf{B}_{a}$ to the spaces $F \otimes \Lambda^{0} \mathbb{C}$ and $F \otimes \Lambda^{1} \mathbb{C}$ respectively. It follows from (1.2) that ind $\mathbf{B}_{a}=0$ for $a \ll 0$ and, if $a \gg 0$, then all the sections in Ker $\mathbf{B}_{a}^{2}$ are concentrated on the cylinder $M \times(0, \infty)$, not far from $M \times\{a\}$ (this part of the proof essentially repeats the arguments of Witten in 11]). Hence, the calculation of $\mathrm{Ker}_{\mathbf{B}}^{2}$ is reduced to a problem on the cylinder $M \times(0, \infty)$. It is not difficult now to show that ind $\mathbf{B}_{a}=$ ind $A$ for $a \gg 0$.

Theorem 1.2 now follows from the fact that ind $\mathbf{B}_{a}$ is independent of $a$.

\section{INDEX OF THE OPERATOR $\mathbf{B}_{a}$}

2.1. Let us consider two anti-commuting actions (left and right action) of the Clifford algebra of $\mathbb{R}$ on the exterior algebra $\Lambda^{\bullet} \mathbb{C}=\Lambda^{0} \mathbb{C} \oplus \Lambda^{1} \mathbb{C}$, given by the formulas

$$
c_{L}(t) \omega=t \wedge \omega-\iota_{t} \omega, \quad c_{R}(t) \omega=t \wedge \omega+\iota_{t} \omega
$$

We will use the notation of Subsection 1.3 In particular, $\tilde{W}$ is the manifold obtained from $W$ by attaching a cylinder, $\tilde{F}=F \otimes \Lambda \bullet \mathbb{C}$ and $\tilde{B}$ is the operator defined in (1.1).

Let $s: \mathbb{R} \rightarrow[0, \infty)$ be a smooth function such that $s(t)=t$ for $|t| \geq 1$, and $s(t)=0$ for $|t| \leq 1 / 2$. Consider the map $p: \tilde{W} \rightarrow \mathbb{R}$ such that $p(y, t)=s(t)$ for $(y, t) \in M \times(0, \infty)$ and $p(x)=0$ for $x \in W$. Define the operator

$$
\mathbf{B}_{a}:=\tilde{B}-1 \otimes c_{R}((p(x)-a)) .
$$

The same proof as in [3, Th. 1.17] shows that the operator $\mathbf{B}_{a}$ is essentially selfadjoint with the initial domain smooth compactly supported sections. We will also denote by $\mathbf{B}_{a}$ the extension of this operator to a self-adjoint operator on the space of square-integrable sections. 
Lemma 2.2. Let $\Pi_{i}: \tilde{F} \rightarrow F \otimes \Lambda^{i} \mathbb{C} \quad(i=0,1)$ be the projections. Then

$$
\mathbf{B}_{a}^{2}=B^{2} \otimes 1-R+|p(x)-a|^{2},
$$

where $R: \tilde{F} \rightarrow \tilde{F}$ is a uniformly bounded bundle map whose restriction to $M \times$ $(1, \infty)$ is equal to $\sqrt{-1} \gamma\left(\Pi_{1}-\Pi_{0}\right)$ and whose restriction to $W$ vanishes.

Proof. Note, first, that $p(x)-a \equiv-a$ on $W$. Thus, since $c_{R}(a)$ anti-commutes with $\tilde{B}$, we have $\left.\mathbf{B}_{a}^{2}\right|_{W}=\left.\tilde{B}^{2}\right|_{W}+a^{2}=\left.B^{2} \otimes 1\right|_{W}+a^{2}$. Hence, (2.3) holds when restricted to $W$.

We now consider the restriction of $\mathbf{B}_{a}^{2}$ to the cylinder $M \times(0, \infty)$. Recall that the function $s: \mathbb{R} \rightarrow[0, \infty)$ was defined in Subsection 2.1. Clearly,

$$
\left.\mathbf{B}_{a}\right|_{M \times(0, \infty)}=\sqrt{-1} A \otimes c_{L}(1)+\sqrt{-1} \gamma \otimes c_{L}(1) \frac{\partial}{\partial t}+(s(t)-a) 1 \otimes c_{R}(1) .
$$

Since the operators $c_{L}$ and $c_{R}$ anti-commute, we obtain

$$
\left.\mathbf{B}_{a}^{2}\right|_{M \times(0, \infty)}=B^{2} \otimes 1+\sqrt{-1} s^{\prime} \gamma \otimes c_{L}(1) c_{R}(1)+|t-a|^{2} .
$$

Since $c_{L}(1) c_{R}(1)=\Pi_{1}-\Pi_{0}$, it follows that equality 2.3 holds with $R=$ $s^{\prime} \sqrt{-1} \gamma\left(\Pi_{1}-\Pi_{0}\right)$.

Lemma 2.3. The spectrum of the operator $\mathbf{B}_{a}$ is discrete.

Proof. It is well known (cf., for example, [10, Lemma 6.3]) that the lemma is equivalent to the following statement: For any $\varepsilon>0$ there exists a compact set $K \subset \tilde{W}$, such that if $u$ is a smooth compactly supported section of $\tilde{F}$, then

$$
\int_{\tilde{W} \backslash K}|u|^{2} d \mu<\varepsilon \int_{\tilde{W}}\left\langle\mathbf{B}_{a}^{2} u, u\right\rangle d \mu .
$$

Here, $d \mu$ is the Riemannian volume element on $\tilde{W}$, and $\langle\cdot, \cdot\rangle$ denotes the Hermitian scalar product on the fibers of $\tilde{F}$.

Set $V(x)=|p(x)-a|^{2}-R$. To prove (2.4) note that, since $R$ is bounded, there exists a compact set $K \subset \tilde{W}$ such that $V>1 / \varepsilon$ on $\tilde{W} \backslash K$. Note, also, that the first summand in (2.3) is a non-negative operator. Hence, we have

$$
\int_{\tilde{W} \backslash K}|u|^{2} d \mu<\varepsilon \int_{\tilde{W} \backslash K}\langle V u, u\rangle d \mu \leq \varepsilon \int_{\tilde{W}}\langle V u, u\rangle d \mu \leq \varepsilon \int_{\tilde{W}}\left\langle\mathbf{B}_{a}^{2} u, u\right\rangle d \mu .
$$

Set $\tilde{F}^{+}:=F \otimes \Lambda^{0} \mathbb{C}, \tilde{F}^{-}:=F \otimes \Lambda^{1} \mathbb{C}, \mathbf{B}_{a}^{ \pm}:=\left.\mathbf{B}_{a}\right|_{\Gamma\left(\tilde{W}, \tilde{F}^{ \pm}\right)}$and define

$$
\operatorname{ind} \mathbf{B}_{a}=\operatorname{dim} \operatorname{Ker} \mathbf{B}_{a}^{+}-\operatorname{dim} \operatorname{Ker} \mathbf{B}_{a}^{-} \text {. }
$$

Lemma 2.4. The index ind $\mathbf{B}_{a}$ is independent of $a$.

Proof. From (2.2), we see that $\mathbf{B}_{b}-\mathbf{B}_{a}=1 \otimes c_{R}(b-a)$ is a bounded operator, depending continuously on $b-a \in \mathbb{R}$. The lemma now follows from the stability of the index of a Fredholm operator; cf., for example, [8, §I.8].

Lemma 2.5. $\operatorname{ind}\left(\mathbf{B}_{a}\right)=0$ for all $a \in \mathbb{R}$.

Proof. By Lemma 2.4, it is to enough prove the proposition for one particular value of $a$. But it follows from Lemma 2.2 that if $a$ is a negative number such that $a^{2}>\sup _{x \in \tilde{W}}\|R(x)\|$, then $\mathbf{B}_{a}^{2}>0$, so that $\operatorname{Ker} \mathbf{B}_{a}^{2}=0$. 
To prove Theorem 1.2 it is enough now to show that ind $\mathbf{B}_{a}=\operatorname{ind} A$. This is done in two steps: first, in Section [3, we construct a "model" operator $\mathbf{B}^{\mathrm{mod}}$ on the cylinder $M \times(-\infty, \infty)$, whose index is equal to ind $A$. Then, in Section 4 we show that ind $\mathbf{B}_{a}=$ ind $\mathbf{B}^{\text {mod }}$.

\section{THE MODEL OPERATOR}

The bundles $E^{ \pm}$lift to Hermitian vector bundles over the cylinder $M \times \mathbb{R}$, which we will denote by the same letters. Consider the Hermitian vector bundle $\tilde{F}:=\left(E^{+} \oplus E^{-}\right) \otimes \Lambda^{\bullet} \mathbb{C}$ and the operator $\mathbf{B}^{\bmod }: C^{\infty}(M \times \mathbb{R}, \tilde{F}) \rightarrow C^{\infty}(M \times \mathbb{R}, \tilde{F})$ defined by

$$
\mathbf{B}^{\mathrm{mod}}:=\sqrt{-1} A \otimes c_{L}(1)+\sqrt{-1} \gamma \otimes c_{L}(1) \frac{\partial}{\partial t}+1 \otimes c_{R}(t)
$$

where $t$ is the coordinate along the axis of the cylinder. We refer to $\mathbf{B}^{\text {mod }}$ as the model operator; cf. [9]. As in Section 2] it is essentially self-adjoint and has discrete spectrum. We define ind $\mathbf{B}^{\bmod }$ by 2.5).

Lemma 3.1. The kernel of the model operator $\mathbf{B}^{\text {mod }}$ is isomorphic (as a graded space) to $\operatorname{Ker}(A)$. In particular, ind $\mathbf{B}^{\bmod }=\operatorname{ind} A$.

Proof. The same calculations as in the proof of Lemma 2.2 show that

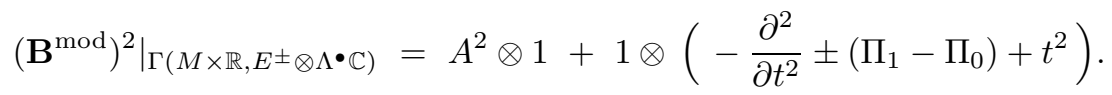

Both summands on the right-hand side of (3.1) are non-negative. Hence, the kernel of $\left(\mathbf{B}^{\text {mod }}\right)^{2}$ is given by the tensor product of the kernels of these operators.

The space $\operatorname{Ker}\left(-\frac{\partial^{2}}{\partial t^{2}}+\Pi_{1}-\Pi_{0}+t^{2}\right)$ is one dimensional and is spanned by the function $\alpha^{+}(t):=e^{-t^{2} / 2} \in \Lambda^{0} \mathbb{R}$. Similarly, $\operatorname{Ker}\left(-\frac{\partial^{2}}{\partial t^{2}}+\Pi_{0}-\Pi_{1}+t^{2}\right)$ is one dimensional and is spanned by the one-form $\alpha^{-}(t):=e^{-t^{2} / 2} d s$, where we denote by $d s$ the generator of $\Lambda^{1} \mathbb{C}$. It follows that

$$
\left.\operatorname{Ker}\left(\mathbf{B}^{\bmod }\right)^{2}\right|_{\Gamma\left(M \times \mathbb{R}, E^{ \pm} \otimes \Lambda^{\bullet} \mathbb{C}\right)} \simeq\left\{\sigma \otimes \alpha^{ \pm}(t):\left.\sigma \in \operatorname{Ker} A^{2}\right|_{\Gamma\left(M, E^{ \pm}\right)}\right\}
$$

3.2. Let $T_{a}: M \times \mathbb{R} \rightarrow M \times \mathbb{R}, T_{a}(x, t)=(x, t+a)$ be the translation and consider the pull-back map $T_{a}^{*}: \Gamma(M \times \mathbb{R}, \tilde{F}) \rightarrow \Gamma(M \times \mathbb{R}, \tilde{F})$. Set

$$
\mathbf{B}_{a}^{\bmod }:=T_{-a}^{*} \circ \mathbf{B}^{\bmod } \circ T_{a}^{*}=B \otimes 1-1 \otimes c_{R}(t-a) .
$$

Then ind $\mathbf{B}_{a}^{\bmod }=$ ind $\mathbf{B}^{\text {mod }}$, for any $a \in \mathbb{R}$.

\section{Proof of Theorem 1.2}

If $A$ is a self-adjoint operator with discrete spectrum and $\lambda \in \mathbb{R}$, we denote by $N(\lambda, A)$ the number of the eigenvalues of $A$ not exceeding $\lambda$ (counting multiplicities).

Let $\mathbf{B}_{a}^{ \pm}$denote the restriction of $\mathbf{B}_{a}$ to the spaces $\Gamma\left(\tilde{W}, \tilde{F}^{ \pm}\right)$. Similarly, let $\mathbf{B}_{ \pm}^{\text {mod }}, \mathbf{B}_{ \pm, a}^{\text {mod }}$ denote the restriction of the operators $\mathbf{B}^{\text {mod }}, \mathbf{B}_{a}^{\text {mod }}$ to the spaces $\Gamma\left(M \times \mathbb{R}, \tilde{F}^{ \pm}\right)$. 
Proposition 4.1. Let $\lambda_{ \pm}$denote the smallest non-zero eigenvalue of $\left(\mathbf{B}_{ \pm}^{\text {mod }}\right)^{2}$. Then, for any $0<\varepsilon<\min \left\{\lambda_{+}, \lambda_{-}\right\}$, there exists $A=A(\varepsilon, V)>0$, such that

$$
N\left(\lambda_{ \pm}-\varepsilon,\left(\mathbf{B}_{a}^{ \pm}\right)^{2}\right)=\operatorname{dim} \operatorname{Ker}\left(\mathbf{B}_{ \pm}^{\text {mod }}\right)^{2} \quad \text { for all } a>A .
$$

Before proving the proposition let us explain how it implies Theorem 1.2.

4.2. Proof of Theorem 1.2. Let $V_{\varepsilon, a}^{ \pm} \subset \Gamma\left(\tilde{W}, \tilde{F}^{ \pm}\right)$denote the vector space spanned by the eigenvectors of the operator $\left(\mathbf{B}_{a}^{ \pm}\right)^{2}$ with eigenvalues smaller than or equal to $\lambda_{ \pm}-\varepsilon$. The operator $\mathbf{B}_{a}^{ \pm}$sends $V_{\varepsilon, a}^{ \pm}$into $V_{\varepsilon, a}^{\mp}$. It follows that

$$
\operatorname{dim} \operatorname{Ker} \mathbf{B}_{a}^{+}-\operatorname{dim} \operatorname{Ker} \mathbf{B}_{a}^{-}=\operatorname{dim} V_{\varepsilon, a}^{+}-\operatorname{dim} V_{\varepsilon, a}^{-} .
$$

By Proposition 4.1 the right-hand side of this equality equals $\operatorname{dim} \operatorname{Ker} \mathbf{B}_{+}^{\bmod }-$ $\operatorname{dim} \operatorname{Ker} \mathbf{B}_{-}^{\text {mod }}$. Thus ind $\mathbf{B}_{a}=$ ind $\mathbf{B}^{\text {mod }}$. Theorem 1.2 now follows from Lemmas 2.5 and 3.1 .

The rest of this section is occupied with the proof of Proposition 4.1.

4.3. Estimate from above on $N\left(\lambda_{ \pm}-\varepsilon,\left(\mathbf{B}_{a}^{ \pm}\right)^{2}\right)$. We will first show that

$$
N\left(\lambda_{ \pm}-\varepsilon,\left(\mathbf{B}_{a}^{ \pm)^{2}}\right)\right) \leq \operatorname{dim} \operatorname{Ker} \mathbf{B}_{ \pm}^{\mathrm{mod}}
$$

To this end we will estimate the operator $\mathbf{B}_{a}^{2}$ from below. We will use the technique of [9, 1, adding some necessary modifications.

4.4. The IMS localization. Let $j, \bar{j}: \mathbb{R} \rightarrow[0,1]$ be smooth functions such that $j^{2}+\bar{j}^{2} \equiv 0$ and $j(t)=1$ for $t \geq 3$, while $j(t)=0$ for $t \leq 2$. Set $j_{a}(t)=$ $j\left(a^{-1 / 2} t\right), \quad \bar{j}_{a}(t)=\bar{j}\left(a^{-1 / 2} t\right)$. These functions induce smooth functions on the cylinder $M \times[0,1]$, which we denote by the same letters. By a slight abuse of notation we will also denote by the same letters the smooth functions on $\tilde{W}$ given by the formulas $j_{a}(x)=j\left(a^{-1 / 2} p(x)\right), \bar{j}_{a}(x)=\bar{j}\left(a^{-1 / 2} p(x)\right)$.

The following version of IMS1 localization formula is due to Shubin [9, Lemma 3.1].

Lemma 4.5. The following operator identity holds:

$$
\mathbf{B}_{a}^{2}=\bar{j}_{a} \mathbf{B}_{a}^{2} \bar{j}_{a}+j_{a} \mathbf{B}_{a}^{2} j_{a}+\frac{1}{2}\left[\bar{j}_{a},\left[\bar{j}_{a}, \mathbf{B}_{a}^{2}\right]\right]+\frac{1}{2}\left[j_{a},\left[j_{a}, \mathbf{B}_{a}^{2}\right]\right] .
$$

Proof. Using the equality $j_{a}^{2}+\bar{j}_{a}^{2}=1$ we can write

$$
\mathbf{B}_{a}^{2}=j_{a}^{2} \mathbf{B}_{a}^{2}+\bar{j}_{a}^{2} \mathbf{B}_{a}^{2}=j_{a} \mathbf{B}_{a}^{2} j_{a}+\bar{j}_{a} \mathbf{B}_{a}^{2} \bar{j}_{a}+j_{a}\left[j_{a}, \mathbf{B}_{a}^{2}\right]+\bar{j}_{a}\left[\bar{j}_{a}, \mathbf{B}_{a}^{2}\right] .
$$

Similarly, $\mathbf{B}_{a}^{2}=\mathbf{B}_{a}^{2} j_{a}^{2}+\mathbf{B}_{a}^{2} \bar{j}_{a}^{2}=j_{a} \mathbf{B}_{a}^{2} j_{a}+\bar{j}_{a} \mathbf{B}_{a}^{2} \bar{j}_{a}-\left[j_{a}, \mathbf{B}_{a}^{2}\right] j_{a}-\left[\bar{j}_{a}, \mathbf{B}_{a}^{2}\right] \bar{j}_{a}$. Summing these identities and dividing by 2 , we come to (4.3).

We will now estimate each of the summands on the right-hand side of (4.3).

Lemma 4.6. There exists $A>0$ such that $\bar{j}_{a} \mathbf{B}_{a}^{2} \bar{j}_{a} \geq \frac{a^{2}}{8} \bar{j}_{a}^{2}$, for all $a>A$.

Proof. Note that $p(x) \leq 3 a^{1 / 2}$ for any $x$ in the support of $\bar{j}_{a}$. Hence, if $a>36$, we have $\bar{j}_{a}^{2}|p(x)-a|^{2} \geq \frac{a^{2}}{4} \bar{j}_{a}^{2}$.

Set $A=\max \left\{36,4 \sup _{x \in \tilde{W}}|R|^{1 / 2}\right\}$ and let $a>A$. Using Lemma 2.2 we obtain

$$
\bar{j}_{a} \mathbf{B}_{a}^{2} \bar{j}_{a} \geq \bar{j}_{a}^{2}|p(x)-a|^{2}-\bar{j}_{a} R \bar{j}_{a} \geq \frac{a^{2}}{8} \bar{j}_{a}^{2} .
$$

\footnotetext{
${ }^{1}$ The abbreviation IMS stands for the initials of R. Ismagilov, J. Morgan, I. Sigal and B. Simon.
} 
4.7. Let $P_{a}: L^{2}(M \times \mathbb{R}, \tilde{F}) \rightarrow \operatorname{Ker} \mathbf{B}_{a}^{\text {mod }}$ be the orthogonal projection. Let $P_{a}^{ \pm}$ denote the restriction of $P_{a}$ to the space $L^{2}\left(M \times \mathbb{R}, \tilde{F}^{ \pm}\right)$. Then $P_{a}^{ \pm}$is a finite rank operator and its rank equals $\operatorname{dim} \operatorname{Ker} \mathbf{B}_{ \pm, a}^{\text {mod }}$. Clearly,

$$
\mathbf{B}_{ \pm, a}^{\bmod }+\lambda_{ \pm} P_{a}^{ \pm} \geq \lambda_{ \pm}
$$

By identifying the support of $j_{a}$ in $M \times \mathbb{R}$ with a subset of $\tilde{W}$, we can and will consider $j_{a} P_{a} j_{a}$ and $j_{a} \mathbf{B}_{a}^{\bmod } j_{a}$ as operators on $\tilde{W}$. Then $j_{a} \mathbf{B}_{a}^{2} j_{a}=j_{a} \mathbf{B}_{a}^{\bmod } j_{a}$. Hence, (4.4) implies the following:

Lemma 4.8. $j_{a} \mathbf{B}_{a}^{ \pm} j_{a}+\lambda_{ \pm} j_{a} P_{a}^{ \pm} j_{a} \geq \lambda_{ \pm} j_{a}^{2}, \quad \operatorname{rk} j_{a} P_{a}^{ \pm} j_{a} \leq \operatorname{dim} \operatorname{Ker} \mathbf{B}_{ \pm}^{\text {mod }}$.

For an operator $A: L^{2}(\tilde{W}, \tilde{F}) \rightarrow L^{2}(\tilde{W}, \tilde{F})$, we denote by $\|A\|$ its norm.

Lemma 4.9. Let $C=2 \max \left\{\max \left\{\left|j^{\prime}(t)\right|^{2},\left|\bar{j}^{\prime}(t)\right|^{2}\right\}: t \in \mathbb{R}\right\}$. Then

$$
\left\|\left[j_{a},\left[j_{a}, \mathbf{B}_{a}^{2}\right]\right]\right\| \leq C a^{-1}, \quad\left\|\left[\bar{j}_{a},\left[\bar{j}_{a}, \mathbf{B}_{a}^{2}\right]\right]\right\| \leq C a^{-1} \quad \text { for all } \quad a>0 .
$$

Proof. From Lemma 2.2 we obtain

$\left|\left[j_{a},\left[j_{a}, \mathbf{B}_{a}^{2}\right]\right]\right|=2\left|j_{a}^{\prime}(t)\right|^{2}=2 a^{-1 / 2}\left|j^{\prime}\left(a^{-1 / 2} t\right)\right|, \quad\left|\left[\bar{j}_{a},\left[\bar{j}_{a}, \mathbf{B}_{a}^{2}\right]\right]\right|=2 a^{-1 / 2}\left|j^{\prime}\left(a^{-1 / 2} t\right)\right|$.

From Lemmas 4.5, 4.8 and 4.9 we obtain the following

Corollary 4.10. For any $\varepsilon>0$, there exists $A=A(\varepsilon, V)>0$ such that, for all $a>A$, we have

$$
\mathbf{B}_{a}^{ \pm}+\lambda_{ \pm} j_{a} P_{a}^{ \pm} j_{a} \geq \lambda_{ \pm}-\varepsilon, \quad \operatorname{rk} j_{a} P_{a}^{ \pm} j_{a} \leq \operatorname{dim} \operatorname{Ker} \mathbf{B}_{ \pm}^{\bmod } .
$$

The estimate (4.2) follows from Corollary 4.10) and the following general lemma 7, p. 270]:

Lemma 4.11. Assume that $A, B$ are self-adjoint operators in a Hilbert space $\mathcal{H}$ such that $\operatorname{rk} B \leq k$ and there exists $\mu>0$ such that $\langle(A+B) u, u\rangle \geq \mu\langle u, u\rangle$ for any $u \in \operatorname{Dom}(A)$. Then $N(\mu-\varepsilon, A) \leq k$ for any $\varepsilon>0$.

4.12. Estimate from below on $N\left(\lambda_{ \pm}-\varepsilon,\left(\mathbf{B}_{a}^{ \pm}\right)^{2}\right)$. To prove Proposition 4.1 it now remains to show that

$$
N\left(\lambda_{ \pm}-\varepsilon,\left(\mathbf{B}_{a}^{ \pm}\right)^{2}\right) \geq \operatorname{dim} \operatorname{Ker} \mathbf{B}_{ \pm}^{\bmod } \equiv \operatorname{dim} \operatorname{Ker} \mathbf{B}_{ \pm, a}^{\bmod } .
$$

Let $V_{\varepsilon, a}^{ \pm} \subset L^{2}(\tilde{W}, \tilde{F})$ denote the vector space spanned by the eigenvectors of the operator $\left(\mathbf{B}_{a}^{ \pm}\right)^{2}$ with eigenvalues smaller or equal to $\lambda_{ \pm}-\varepsilon$. Let $\Pi_{\varepsilon, a}^{ \pm}: L^{2}\left(\tilde{W}, \tilde{F}^{ \pm}\right) \rightarrow$ $V_{\varepsilon, a}^{ \pm}$be the orthogonal projection. Then $\operatorname{rk} \Pi_{\varepsilon, a}^{ \pm}=N\left(\lambda_{ \pm}-\varepsilon,\left(\mathbf{B}_{a}^{ \pm}\right)^{2}\right)$. As in Subsection 4.7 we can and will consider $j_{a} \Pi_{\varepsilon, a}^{ \pm} j_{a}$ as an operator on $L^{2}\left(M \times \mathbb{R}, \tilde{F}^{ \pm}\right)$. The proof of the following lemma does not differ from the proof of Corollary 4.10

Lemma 4.13. For any $\varepsilon>0$, there exists $A=A(\varepsilon, V)>0$ such that, for any $a>A$, we have

$$
\mathbf{B}_{ \pm, a}^{m o d}+\lambda_{ \pm} j_{a} \Pi_{a}^{ \pm} j_{a} \geq \lambda_{ \pm}-\varepsilon, \quad \operatorname{rk} j_{a} \Pi_{a}^{ \pm} j_{a} \leq \operatorname{dim} N\left(\lambda_{ \pm}-\varepsilon,\left(\mathbf{B}_{a}^{ \pm}\right)^{2}\right) .
$$

The estimate (4.7) now follows from Lemmas 4.13 and 4.11 .

The proof of Proposition 4.1 is complete. 


\section{ACKNOWLEDGEMENTS}

Some of the ideas used in this paper I have learned from John Roe. I would like to thank him for very useful and stimulating discussions.

\section{REFERENCES}

1. M. Braverman and M. Farber, Novikov type inequalities for differential forms with nonisolated zeros, Math. Proc. Cambridge Philos. Soc. 122 (1997), 357-375. MR 99b:58220

2. H.L. Cycon, R.G. Froese, W. Kirsch, and B. Simon, Schrödinger operators with applications to quantum mechanics and global geometry, Texts and Monographs in Physics, Springer-Verlag, 1987. MR 88g:35003

3. M. Gromov and B. Lawson, Positive scalar curvature and the Dirac operator on complete Riemannian manifolds, Inst. Hautes Études Sci. Publ. Math. (1983), no. 58, 295-408. MR 85g:58082

4. N. Higson, A note on the cobordism invariance of the index, Topology 30 (1991), no. 3, 439-443. MR 92f:58171

5. M. Lesch, Deficiency indices for symmetric Dirac operators on manifolds with conic singularities, Topology 32 (1993), no. 3. MR 94e:58133

6. L. I. Nicolaescu, On the cobordism invariance of the index of Dirac operators, Proc. Amer. Math. Soc. 125 (1997). MR 97j:58148

7. M. Reed and B. Simon, Methods of modern mathematical physics IV: Analysis of operators, Academic Press, London, 1978. MR 58:12429c

8. M. Shubin, Pseudodifferential operators and spectral theory, Springer-Verlag, Berlin, New York, 1980. MR 88c:47105

9. - Semiclassical asymptotics on covering manifolds and Morse inequalities, Geom. Funct. Anal. 6 (1996), 370-409. MR 97i:58171

10. S Spectral theory of the Schrödinger operators on non-compact manifolds: qualitative results, Spectral theory and geometry (Edinburgh, 1998), Cambridge Univ. Press, Cambridge, 1999, pp. 226-283. MR 2001d:58037

11. E. Witten, Supersymmetry and Morse theory, J. of Diff. Geom. 17 (1982), 661-692. MR 84b:58111

Department of Mathematics, Northeastern University, Boston, Massachusetts 02115

E-mail address: maxim@neu.edu 